\title{
Tethered Spinal Cord due to Thoracic Spinal Cord Lipoma: Minimally Invasive Surgical Management Case Report and Literature Review
}

\author{
Nasser K Yaghi ${ }^{1}$, David Mazur-Hart ${ }^{1}$, Robert Bodor ${ }^{2}$, Karan Rai $^{1}$, Darius Amjadi ${ }^{3}$, Jeffrey M Pollock ${ }^{2}$, \\ Nathan R Selden', Donald A Ross ${ }^{1,4}$ \\ ${ }^{1}$ Departments of Neurological Surgery, Diagnostic Radiology, ${ }^{2}$ Oregon Health \& Science University, Portland, Oregon; ${ }^{3}$ Pathology \\ Laboratory Services, and ${ }^{4}$ Operative Care Division, Portland Veterans Administration, Portland, Oregon
}

Corresponding Author:

Donald A Ross, MD

Neurological Surgery, Oregon Health \& Science University, Mail Code CH8N 3303 South Bond Avenue, Portland Oregon 97239

Tel: $+503-494-4314$

Fax: $+503-346-3610$

Email: rossdo@ohsu.edu

Received: November 14, 2020

Revised: March 19, 2021

Accepted: April 04, 2021
An unusual case of a thoracic spine lipoma presenting with profound progressive numbness along with difficult to interpret preoperative imaging is discussed. A uniquely minimally invasive surgical treatment approach with successful outcome and improved neurologic symptoms is presented. A literature review and discussion of the benefits and limitations of a minimally invasive surgical technique are provided. A male presented with several months of progressive bilateral lower extremity numbness that ascended to the mid-thoracic spine. Spine magnetic resonance imaging demonstrated a $9 \mathrm{~mm}$ intradural, thoracic spinal mass, which was thought preoperatively to represent an arachnoid cyst with an adhesion or a localized dural ectasia. Subsequent imaging demonstrated a band at the cranial margin of the mass appearing to tether the spinal cord to the dorsal-lateral spinal canal without an arachnoid cyst or osseous defect. Surgical exploration revealed an intradural exophytic, intramedullary fatty mass tethering the spinal cord to the dorsolateral dura. An abnormal patch of dura was observed overlying the fatty attachment but no dural defect was identified. Pathology demonstrated fragments of fibroconnective tissue, scattered mature adipocytes, and entrapped meningeal cells, yielding the diagnosis of a spinal cord lipoma. Follow up imaging demonstrated no residual tethering of the spinal cord.

Key Words: Spine, Lipoma, Tethered spinal cord syndrome, Minimally invasive surgical procedures, Microdissection

\section{INTRODUCTION}

Tethering of the spinal cord can occur in a number of settings, including post-traumatic, post-surgical, herniation through dural defects or in the presence of congenital anomalies ${ }^{4,16)}$.

Spinal lipomas are thought to be a result of failed primary neurulation ${ }^{11)}$. They are some of the most common pathologies encountered in pediatric neurosurgery, however, intradural lipomas that are not associated with bony dysraphism (other than simple filar lipomas) are rare, representing less than $1 \%$ of all intra dural tumors ${ }^{1,3,5-7)}$. Of this group, lipomas located in the thoracic spine are exceedingly rare. Intramedullary lipomas have a bimodal distribution of presentation, first during infancy or early childhood and secondarily in the third to fourth decades of life ${ }^{15)}$.

Lipomas typically cause neurologic deficit as a result of their mass effect, behaving similarly to spinal cord tumors ${ }^{9}$. Additio- nally, spinal lipomas cause neurologic deficit by tethering, in which symptoms arise due to traction longitudinally on the spinal cord $^{16)}$. There is some research to suggest the pathophysiology of tethered cord syndrome is secondary to reduced blood flow, impaired oxidative metabolism, and hypoxia associated with elasticity of the spinal cord $^{2)}$.

The case described here is the first report to our knowledge, to detail an unusual occurrence of a symptomatic tethered thoracic spinal cord due to an intra- and extra-medullary lipoma that was difficult to diagnose preoperatively. Subsequently, the case was managed successfully with minimally invasive surgery. A literature review was also conducted.

\section{Literature Analysis}

A literature search of the PubMed database was performed using the algorithm ["spine" OR "spinal" AND "lipoma" AND "mini- 
mally invasive"] up to March 2021. Article titles and abstracts were then individually screened for relevance related to thoracic lipomas.

\section{CLINICAL PRESENTATION}

\section{Ethical Approval}

With Institutional Review Board approval with waiver of consent, a chart review was conducted on this novel case.

\section{Case Report}

The patient is a 26-year-old male with a history of chronic low back pain that was first evaluated in a neurology outpatient clinic. There was a three-and-a-half-week history of insidious onset bilateral lower extremity ascending numbness. Sensory deficit was bilaterally symmetric, constant, and did not change with activity or position. He had sustained an 80 foot fall down a mountain while serving in the military approximately 5 years prior, but denied recent trauma or falls. He subsequently reported
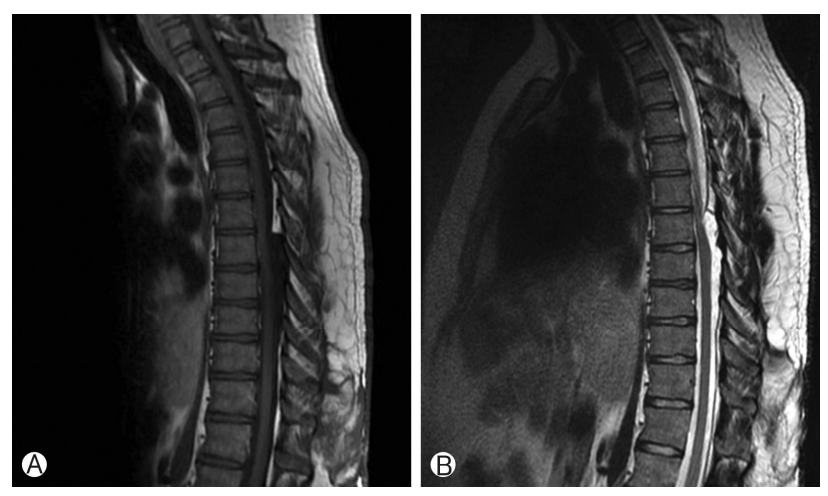

Figure 1. Sagittal T1-weighted MRI (A) Shows a hypointense mass lesion in the dorsal spinal canal extending from T6-T9. Sagittal T2weighted MRI (B) Shows the lesion is hyperintense, with a poorly defined inferior margin. involvement of his bilateral upper extremities with numbness in his fingers. There was no significant bowel or bladder incontinence, nor saddle anesthesia. There was no family history of neuromuscular disorders or multiple sclerosis.

His neurological examination was significant for complete loss of sensation to light touch and reduced vibration sense from the level of waist down bilaterally. He had intact deep pressure sensation and proprioception. His strength was full in all muscle groups. His patellar reflexes were $3+$ bilaterally without clonus, Hoffman's sign, nor Babinski's sign. Diagnostic electromyography (EMG) and a nerve conduction study (NCS) showed absent sural and superficial peroneal nerve sensory responses suggestive of peripheral polyneuropathy that was length-dependent. The upper limb sensory responses were preserved, with mild bilateral carpal tunnel syndrome. Of note, he had had an EMG of the bilateral lower extremities 2 years prior, which was normal. Extensive blood work was unrevealing. Cervical and lumbar spine magnetic resonance imaging (MRI) were unremarkable. Chest, abdomen, and pelvis computed tomography $(\mathrm{CT})$ revealed no obvious signs of malignancy. There was evidence of a thickened terminal ileum and rectum for which the patient had undergone an endoscopy with negative biopsies.

Thoracic spine MRI showed a T1-hypointense, T2-hyperintense, non-enhancing lesion in the intradural, extramedullary space of the dorsal spinal canal at T6-T9, measuring $9 \mathrm{~mm}$ in anterior-posterior diameter (Figure 1A, B). This lesion had resulted in ventral displacement of the spinal cord, and appeared to be associated with absence of the posterior epidural fat (Figure 2A). The lesion did not restrict diffusion. At the superior margin of the lesion, there was a thin T2-hypointense, T1-hyperintense band of tissue thought to represent a fibrous adhesion, which extended through the dorsal lesion and appeared to tether the spinal cord to the left dorsal-lateral aspect of the spinal canal (Figure 2B, C). Subsequent CT myelogram demonstrated contiguity of the dorsal intraspinal lesion inferiorly with the thecal sac, multilevel lobular dorsal expansion of the thecal sac, transient flow limitation at the superior margin in the region of the fibrous tissue band, and tethering of the thoracic spinal cord to the left dorsal-lateral surface of the thecal sac (Figures $3 A-E$ ).
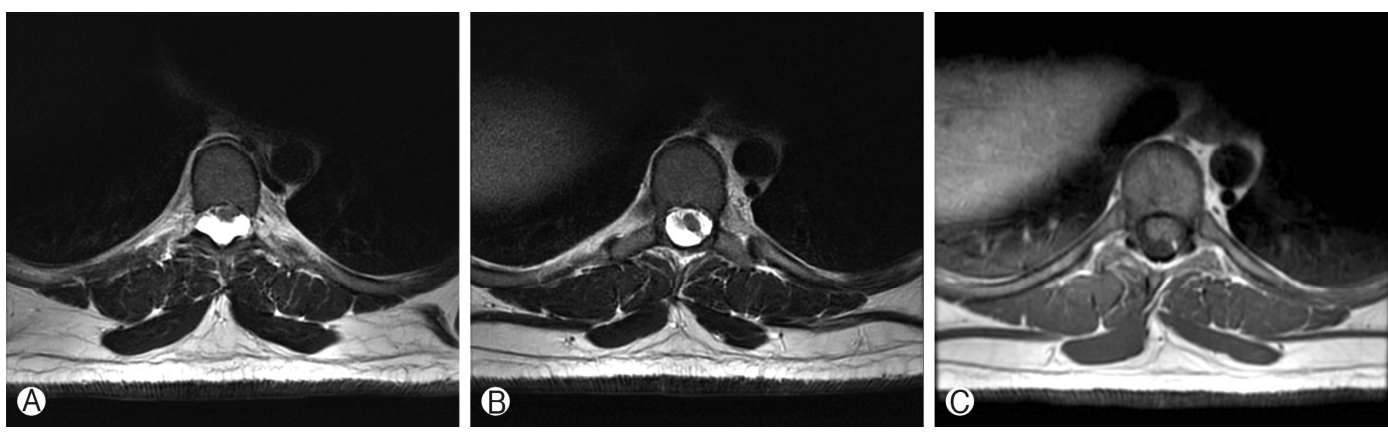

Figure 2. Axial T2-weighted MRI (A) Shows ventral displacement of the spinal cord due to the hyperintense dorsal spinal lesion. At the superior margin of the lesion, axial T2-weighted MRI (B) Shows a linear hypointense fibrous band of tissue which is hyperintense on axial T1-weighted MRI (C) and appears to traverse the dorsal lesion, extending from the dura of the spinal cord to the left dorsal-lateral margin of the spinal canal. 
Ultimately, after review by multiple neuroradiologists, these findings were thought to represent a form of dural ectasia extending into the epidural fat, resulting from altered cerebrospinal fluid (CSF) flow dynamics created by the fibrous band. The patient was indicated for surgical exploration and spinal cord detethering due to progressive neurological deficits.

\section{Surgical Technique}

Surgical exploration was carried out by a minimally invasive, unilateral, trans-muscular, paraspinal approach using the Quadrant retractor (Medtronic, Dublin, Ireland) exposing spine levels T6 to T8. The left hemilaminae and ipsilateral medial facets of
T6 to T8 and the base of the spinous processes were removed. The ligamentum flavum was dissected and removed. Underneath, an abnormally thin and unusual appearing, but apparently intact, dura was encountered. Nevertheless, scant amounts of CSF were observed entering the field. Rostrally and caudally the abnormal appearing dura had a bluish and ballotable appearance. However, in the central area of the decompression, dura had a yellowish, ovoid appearance, which to palpation was solid. A small opening in what was suspected to be dura just medial to this firm, yellowish area was made and the subarachnoid space entered, where brisk CSF drainage was encountered and the right lateral aspect of the spinal cord was visualized. The spinal cord was observed to be tethered to a yellowish mass in the dura by a dorsal band
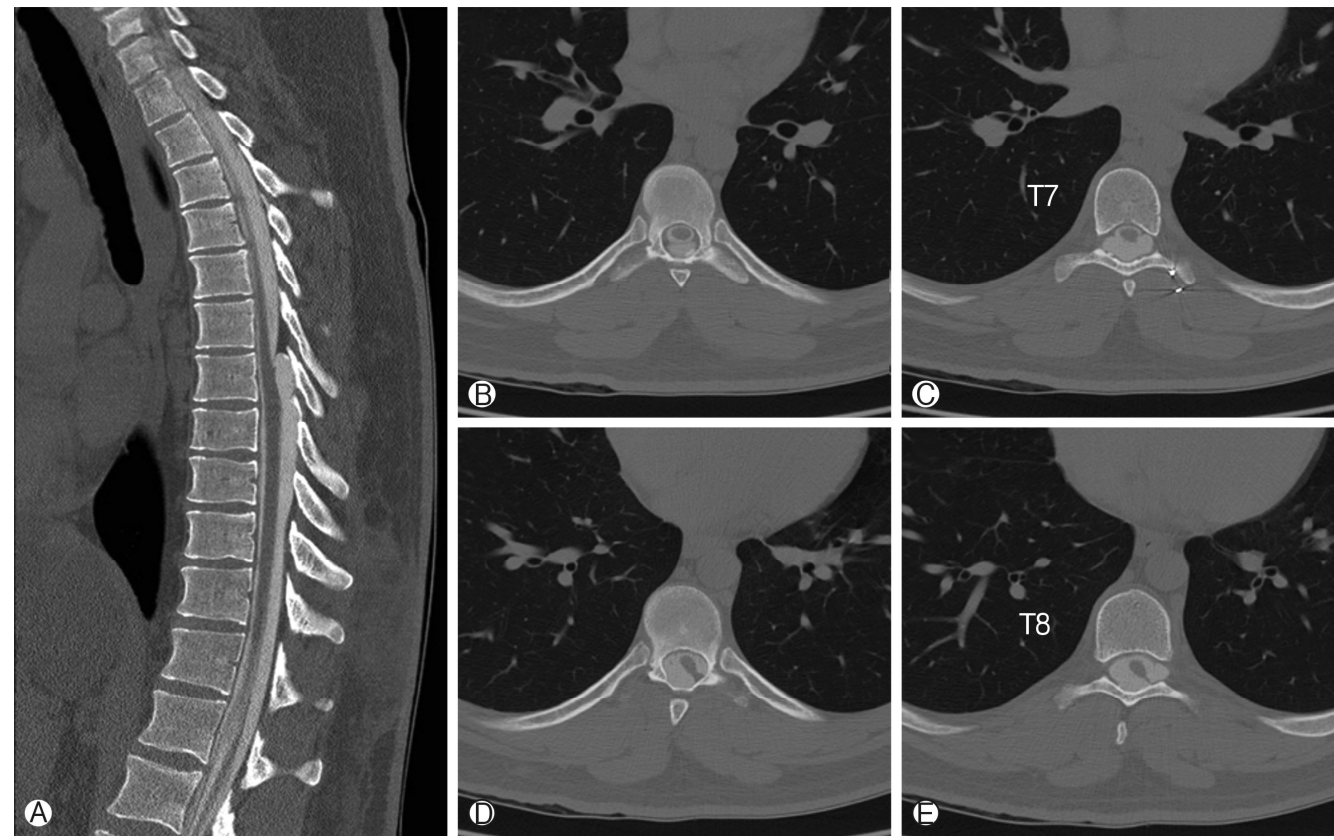

Figure 3. Sagittal CT myelogram (A) shows that the dorsal spinal lesion is contiguous with the thecal sac inferiorly, filling briskly with contrast. During injection, there was temporary flow limitation at the superior margin of the lesion at T6-T7, thought to be related to the fibrous band in this area. At T6-T7 on Axial CT myelogram (B) There is an unusual bi-lobed appearance of the thecal sac thought to be due to the fibrous band. Moving caudally on axial imaging the thecal sac has an uncharacteristic non-ovoid shape at $7 \mathbb{C}$, and further caudal at T-T8 interspace (D) and at the T8 vertebral body (E) There is extension of the thoracic spinal cord toward the left dorsal-lateral surface of the thecal sac.
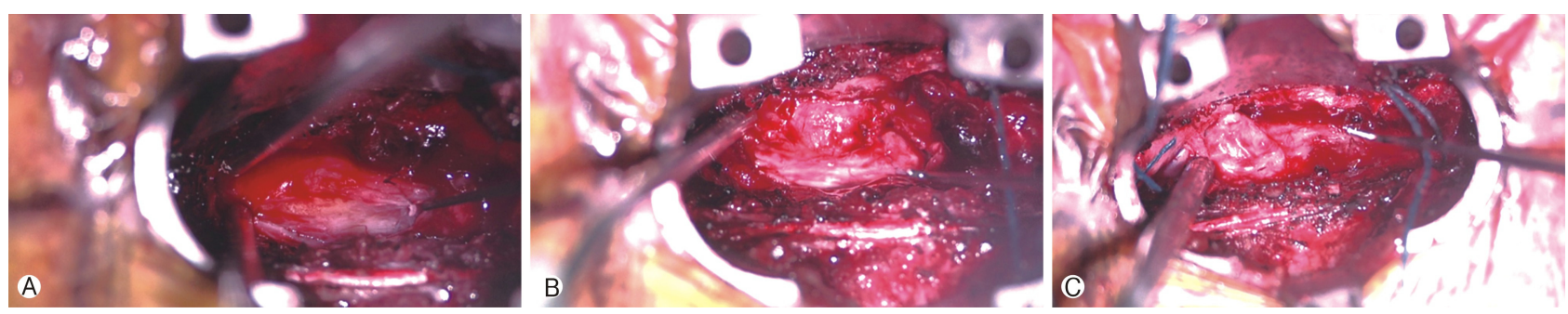

Figure 4. Intraoperative photos showing initial exposure of thecal sac at T6-T7 via Quadrant retractor and medial dura opening exposing a yellow lipomatous mass (A). The dura opened circumferentially around the mass with thoracic spinal cord ventral to presumed placode/ lipoma (B). After careful microdissection, we had the placode/ lipoma entirely free of the dura aside from a central stalk and the placode/ lipoma had a mushroom shape (C). 


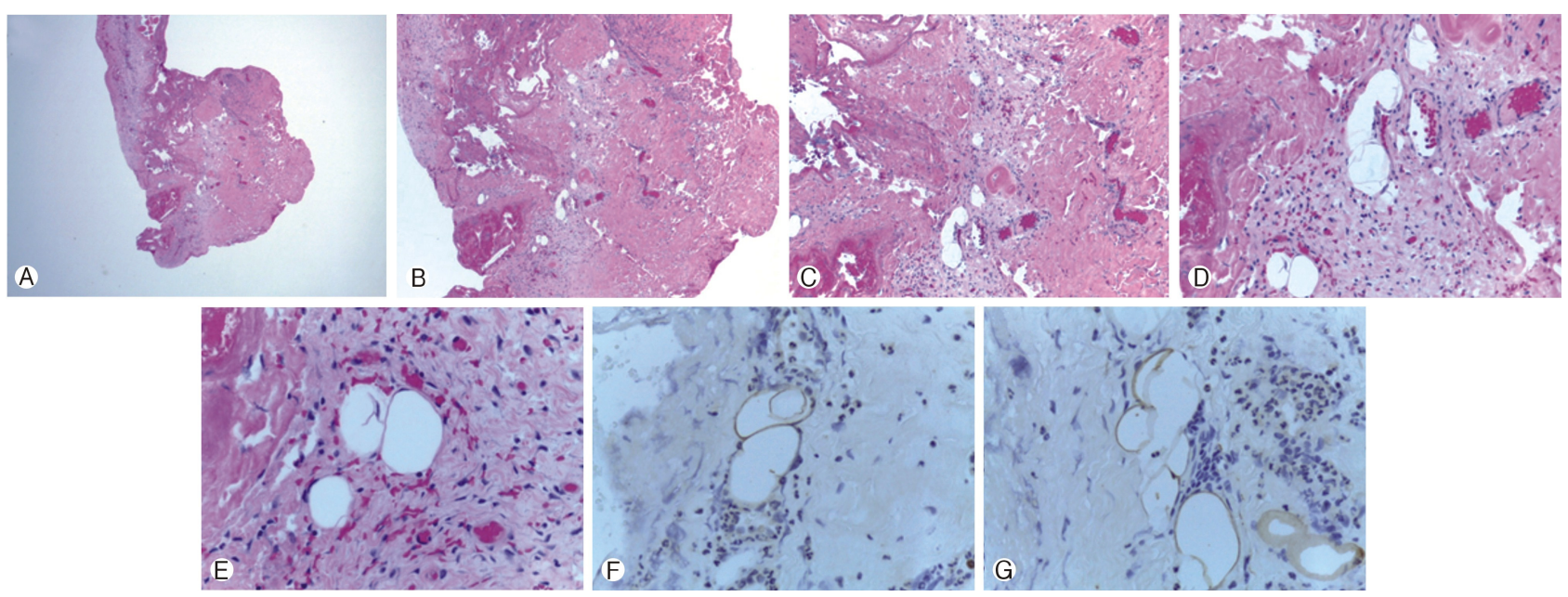

Figure 5. Fragments of fibroconnective tissue with chronic inflammation, focal scattered mature adipocytes, and entrapped meningeal cells. No neural tissue identified. (A) Low powered, (B) $50 \times$, (C) $100 \times$, (D) $200 \times$, (E) $400 \times$, (F) $400 \times$ S100 stain, (G) $400 \times$ S100 stain.

of adipose tissue (Figure 4A). While similar to a narrow, paramedian dorsal lipomyelomeningocele, the fatty mass did not extend through the dura nor was there any bifid dorsal bony structure appreciated. A small biopsy of this area was sent for frozen section and subsequent pathology analysis.

A microsurgical technique to open the dura circumferentially around the attachment of the fatty mass (Figure 4B), was used; very carefully opening the arachnoid, and separating the mass and its overlying dura from the surrounding, intact dura, but leaving an island of abnormal dura on the dorsal surface of the mass. Nerve roots on the patient's left side were identified and preserved. After a period of careful microdissection, the mass was entirely free of the dura with a circumferential dissection around it. The mass was observed to be mushroom shaped (Figure $4 \mathrm{C})$, and resection of the flanges of this mushroom was undertaken while leaving the central area, which arose directly from the dorsal spinal cord intact. Neuro-monitoring was not available to ensure that the dorsal columns of the spinal cord were not entered. The resected tissue was submitted to pathology for analysis (Figures $5 \mathrm{~A}-\mathrm{G}$ ).

At closure, a dural substitute (Biodesign, Cook Medical, Bloomington, IN) was used. It was trimmed to a shape that would fit intradurally inside the dural defect and tucked underneath the dural edges so that CSF pressure would aid in closing the defect. The tenuous dura was not deemed appropriate for direct suture repair. This onlay closure was sealed with fibrin glue. The bony defect created by the approach was then closed with $5 \mathrm{~mL}$ of HydroSet hydroxyapatite bone cement (Stryker, Kalamazoo, MI) over the dural repair.

\section{Post-Operative Management}

The patient woke from anesthesia with significant improvement in his bilateral lower extremity sensation. The patient was managed postoperatively in three degrees of Trendelenburg for 48 hours. He was allowed to sit up, and he was discharged on

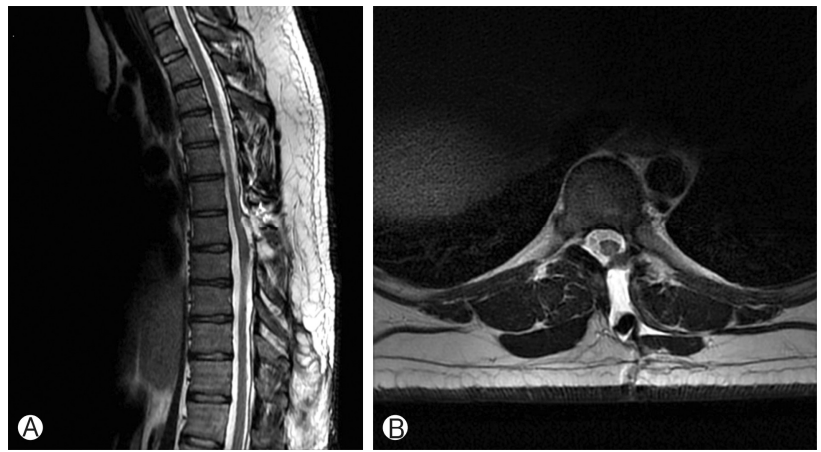

Figure 6. Two month follow-up MRI shows post-surgical changes of resection of the dorsal intraspinal mass on sagittal T2-weighted MRI (A) and axial T2-weighted MRI (B). On axial T2-weighted MRI (B) the spinal cord abuts the left dorsolateral aspect of the thecal sac. A fluid collection extends from the dural surface to the left dorsal paraspinal musculature, also seen on sagittal T2-weighted MRI.

postoperative day three. He was subsequently seen in clinic for a 2-week follow up visit at which time his bilateral lower extremity numbness had resolved completely. He also reported that his legs felt "hyper-sensitive." At a 3-month postoperative follow up, he reported the numbness in his legs, gait difficulty, and hypersensitivity had resolved. He reported no headaches.

Post-operative MRI revealed untethering of the spinal cord with a small, infra-fascial pseudomeningocele (Figure 6A, B). No CSF leak was noted at any time. The patient will follow up with 1-year surveillance MRI.

\section{Literature Review}

Review of the minimally invasive spine surgery literature yielded no published cases with positively identifying features, similar to the case presented here. Our literature search yielded only five manuscripts, of which none were related to thoracic lipomas. 


\section{DISCUSSION}

We report a case of a successful uniquely minimally invasive surgical treatment approach of a rare spine lipoma. This intradural, mixed intra- and extra-medullary mass presented here proved difficult to characterize by imaging. Despite the lipomatous component, it did not display prominent fat-signal on T2-weighted imaging. MRI findings initially suggested an arachnoid cyst. The fibrous adhesions and contiguity of the lesion with the thecal sac on myelography suggested the lesion more closely resembled dural ectasia. The final pathologic and intraoperative findings of a true spinal cord lipoma with tethering, lacking the more extensive dysraphic features of a lipomyelomeningocele, demonstrate a rare presentation of an even more rare entity.

There is a growing belief among surgeons that aggressive total resection of intradural lipomas is associated with poor out come and not required for neurologic improvements). Though controversial, there is some evidence to suggest there is a high rate of recurrence, roughly $50 \%$ with partial resection at 2-10 years postoperativley, though many of these studies analyzed outcomes in patients with lumbosacral lipomyelomeningoceles, which may have a different natural history than presented in this rare case report ${ }^{12,13)}$.

One of the major challenges to surgical resection of spinal lipomas is distinguishing normal spinal cord tissue from lipoma. The minimally invasive approach to spine surgery as undertaken through tubular retractors was highly favorable in this case. This approach allowed for performance of a three level hemilaminectomy to span in the entire area of pathology in the rostro-caudal plane. Visualization of the dura was possible, which was unusually thin, including the raised yellowish-ovoid area that delineated the underlying attachment of the intradural lipoma which identified an appropriate biopsy site. Using a microsurgical technique, separation of the lipoma's dural attachment circumferentially from surrounding normal thoracic spinal dura, thereby untethering the spinal cord, and subsequently relieving the patient's sensory deficits was possible. Others have reported minimally invasive approaches for intradural lesions, but not previously for congenital thoracic spinal lipomas, to our knowledge ${ }^{10)}$.

Given that the thoracic lipoma attached to the inside surface of the abnormal dorsal thoracic dura, which was resected, repair of the resulting large complex opening in a watertight fashion was not possible. Alternatively, a dural substitute was inset to fit the dural defect and sealed with fibrin glue. Given the lack of a water tight dural closure, the minimally invasive tubular approach, which minimizes disruption of soft tissue and muscle, was highly important to ensure minimal risk of CSF leak compared to a traditional open approach. In this patient, while there was an expected post-operative pseudomeningocele, there was no CSF leak or infection. The literature supports this approach and its associated benefits, including reduced rates of surgical site infection and symptomatic CSF leak, along with shorter post-operative hospitalization time ${ }^{14)}$.

Although the tenuous nature of the dura did not allow dural suture closure in this case, suturing of the dura is quite feasible with tubular access surgery using bayoneted instruments and single throw sutures or clips. Open access would have allowed a laminoplasty approach, but otherwise would have major disadvantages, including more postoperative pain, more difficulty with prevention of CSF leakage, higher risk of wound infection, and more blood loss ${ }^{8}$. We believe the potential associated complications of the open spinal approach would outweigh its overall benefits and therefore selected a minimally invasive tubular approach for this unique and rare pathology.

\section{CONCLUSION}

This case of a thoracic spinal cord lipoma not related to bony spinal dysraphism was challenging to identify on pre-operative imaging, and ultimately was treated uniquely with a minimally invasive tubular surgical approach. A rare unusual pattern of imaging findings associated with thoracic spinal lipoma are presented. Feasibility of surgical excision of such a spinal lipoma with untethering through a minimally invasive tubular approach is demonstrated and found to be effective.

\section{CONFLICT OF INTEREST}

No potential conflict of interest relevant to this article.

\section{REFERENCES}

1. Chen K-Y, Osorio J, Rivera J, Chou D: Intramedullary and Extramedullary Thoracic Spinal Lipomas Without Spinal Dysraphism: Clinical Presentation and Surgical Management. World Neurosurg 121:156-159, 2019

2. Filippidis AS, Kalani MY, Theodore N, Rekate HL: Spinal cord traction, vascular compromise, hypoxia, and metabolic derangements in the pathophysiology of tethered cord syndrome. Neurosurg Focus 29:E9, 2010

3. Fujiwara F, Tamaki N, Nagashima T, Nakamura M: Intradural Spinal Lipomas Not Associated with Spinal Dysraphism. Neurosurgery 37:1212-1215, 1995

4. Henry A, Tunkel R, Arbit E, Ku A, Lachmann E: Tethered thoracic cord resulting from spinal cord herniation. Arch Phys Med Rehabil 78:530-533, 1997

5. Ikeda N, Odate S, Shikata J, Yamamura S, Kawaguchi S: Surgical strategies and outcomes for intradural lipomas over the past 20 years. J Clin Neurosci 60:107-111, 2019

6. Klekamp J, Fusco M, Samii M: Thoracic Intradural Extramedullary Lipomas. Report of Three Cases and Review of the Literature. Acta Neurochir (Wien) 143:767-774, 2001

7. Lee M, Rezai AR, Abbott R, Coelho DH, Epstein FJ: Intramedullary spinal cord lipomas. J Neurosurg 82:394-400, 1995

8. Mazur-Hart DJ, Than KD: Minimally Invasive Advances in Deformity. Neurosurg Clin N Am 31:111-120, 2020

9. McGillicuddy GT, Shucart W, Kwan ESK: Intradural Spinal Lipomas. Neurosurgery 21:343-346, 1987

10. Mobbs RJ, Sheldrick K, Phan K: Technical note: Unilateral hemi- 
laminotomy for intradural lesions (UHIL). J Spine Surg 3:71-72, 2017

11. Morota N, Ihara S, Ogiwara H: New classification of spinal lipomas based on embryonic stage. J Neurosurg Pediatr 19:428-439, 2017

12. Pang D: Surgical management of complex spinal cord lipomas: How, why, and when to operate. A review. J Neurosurg Pediatr 23:537-556, 2019

13. Pang D: Total Resection of Complex Spinal Cord Lipomas: How, Why, and When to Operate? Neurol Med Chir (Tokyo) 55:695-
721, 2015

14. Ross DA: Complications of minimally invasive, tubular access surgery for cervical, thoracic, and lumbar surgery. Minim Invasive Surg 2014:451637-451637, 2014

15. Srinivasan US, Raghunathan N, Radhi L: Long term outcome of non-dysraphic intramedullary spinal cord lipomas in adults: Case series and review. Asian Spine J 8:476-483, 2014

16. Theodore N: Novel Concepts in Spine Pathophysiology. Spine (Phila Pa 1976) 41:S25-S26, 2016 